\title{
Condroblastoma de escafoides carpiano
}

\section{Chondroblastoma of the carpal scaphoids}

\author{
F.J. Repáraz ${ }^{1}$, J. Garbayo ${ }^{1}$, M. A. Arrechea ${ }^{2}$, C. Corchuelo ${ }^{1}$, A. Tejero ${ }^{1}$, H. Ayala ${ }^{1}$
}

\section{RESUMEN}

El condroblastoma es un tumor benigno de origen cartilaginoso que representa menos del 1\% de todos los tumores óseos primarios. Se localiza típicamente en la epífisis de los huesos largos. Un 10\% de los condroblastomas afectan a huesos del pie y de la mano. El asiento de esta lesión en el carpo es extraordinariamente raro.

Se presenta un caso de condroblastoma del escafoides del carpo que fue tratado mediante curetaje de la lesión y relleno con injerto óseo autólogo de cresta iliaca.

Palabras clave. Condroblastoma. Escafoides.

\begin{abstract}
A chondroblastoma is a benign tumour of cartilaginous origin which represents less than $1 \%$ of all primary osseous tumours. It is typically localised in the epiphysis of the long bones. Some $10 \%$ of chondroblastomas affect the bones of the foot and the hand. It is extremely rare for the seat of this lesion to be in the carpal bones.

We present a case of chondroblastoma of the carpal scaphoids that was treated through curretage of the lesion and filling with autologous osseous graft from the iliac crest.
\end{abstract}

Palabras clave. Chondroblastoma. Scaphoids.
1. Servicio de Cirugía Ortopédica y Traumatología. Hospital de Navarra. Pamplona.

2. Servicio de Anatomía Patológica. Hospital de Navarra. Pamplona.

Recepción el 20 de junio de 2008

Aceptación provisional el 3 de julio de 2008

Aceptación definitiva el 3 de septiembre de 2008

\section{Correspondencia:}

Francisco Javier Repáraz Padrós

Servicio de Cirugía Ortopédica y Traumatología Hospital de Navarra

Irunlarrea, 3

31008 Pamplona

Tfno. 848422206

E-mail: repamapr@hotmail.com 


\section{INTRODUCCIÓN}

El condroblastoma es una lesión benigna relativamente rara, que fue descrita por Jaffe y Lichtenstein en 1942, específicamente como una entidad clinicopatológica distinta de las llamadas variantes del tumor de células gigantes del hueso ${ }^{1}$. Representa menos del 1\% de los tumores óseos primarios y un $9 \%$ de los benignos. Típicamente se localiza en la epífisis de los huesos largos. El húmero proximal, el fémur distal y la tibia proximal son los lugares afectados más frecuentemente ${ }^{2}$. Un $10 \%$ de los condroblastomas se localizan en los huesos de la mano y el pie, siéndo el astrágalo y el calcáneo los más afectados ${ }^{3}$. La afectación del carpo es excepcionalmente rara $^{2,4}$.

La mayoría de los pacientes tiene menos de 30 años, con un pico de incidencia en la segunda década de la vida y es dos veces más frecuente en hombres ${ }^{2}$.
El comportamiento de estas lesiones es benigno en la gran mayoría de los casos pero hay descritos casos aislados de condroblastoma con transformación maligna espontánea ${ }^{5}$.

El tratamiento habitual de estas lesiones es el curetaje seguido de relleno de la cavidad con hueso esponjoso.

\section{CASO CLÍNICO}

Se presenta un caso de una paciente de 26 años que acude a consulta por dolor en la muñeca derecha de 7 años de evolución sin antecedente traumático. El dolor en reposo era de carácter leve, pero aumentaba con la actividad manual y especialmente con la extensión forzada de la muñeca. No mejoraba con tratamiento médico sintomático.

La interlínea articular radiocarpiana dorsal y el polo proximal del escafoides eran dolorosos a la palpación. No se apreciaba tumoración palpable. La movilidad de la muñeca presentaba

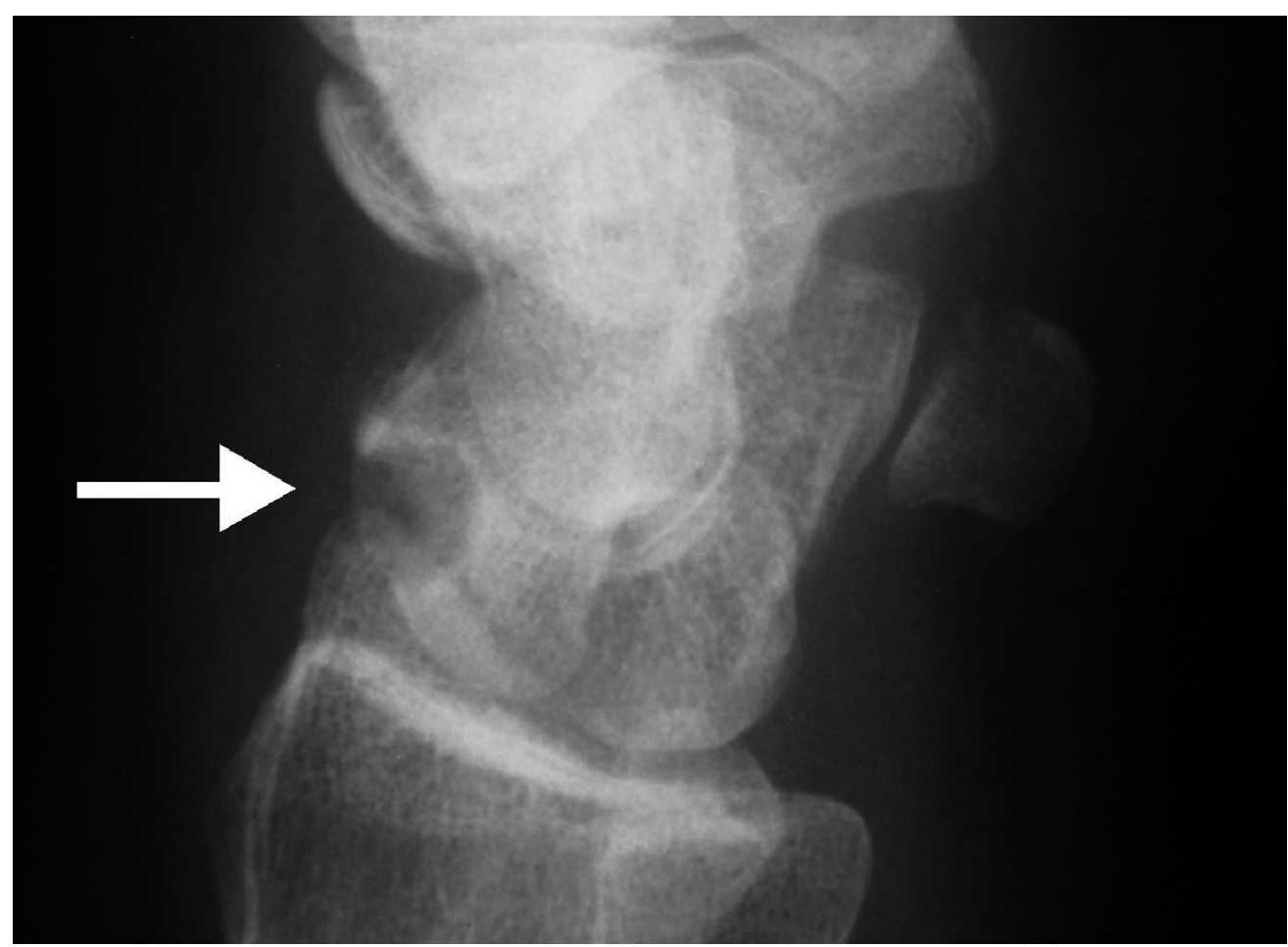

Figura 1. Radiografía en proyección oblicua de la muñeca que muestra una lesión radiolucente, excéntrica en el tercio medio del escafoides. 
una ligera limitación en los últimos grados de flexión y extensión.

La imagen radiológica mostraba una lesión radiolucente, bien definida, ovalada, localizada en el tercio medio-dorsal del escafoides (Fig. 1). En la TAC se apreciaba la localización excéntrica dorsal de la lesión y un punteado compatible con calcificaciones. En la RM destacaban signos de marcado edema óseo en el escafoides, excepto en su tercio distal y sinovitis adyacente. Con el diagnóstico de sospecha de una lesión benigna (encondroma, ganglión intraóseo) se decidió el tratamiento quirúrgico de la lesión. Éste se realizó mediante una vía de abordaje dorsal del escafoides. Tras la apertura de la cápsula articular se expuso la cara dorsal de la epífisis distal del radio y del escafoides, apreciándose en el tercio medio de este último una tumoración de aspecto nacarado y coloración rojo vinosa que ocupaba la cresta dorsal del hueso navicular (Fig. 2). Se realizó un legrado exhaustivo de la lesión y relleno del defecto con injerto óseo esponjoso de cresta iliaca.

El material remitido estaba formado por islotes de matriz cartilaginosa rodeados por un tejido celular abundante constituido por células pseudo-condroblásticas. Dichas células eran redondeadas u ovoides con núcleos hipercromáticos, un citoplasma claro o moderadamente eosinófilo y una membrana plasmática nítida (Fig. 3). El pleomorfismo celular era mínimo y el índice mitótico muy bajo. Algunas células contenían hierro. Entre las células pseudocondroblásticas se encontraban abundantes células gigantes de tipo osteoclástico. En algunas áreas había zonas de calcificación irregular. Se observaban además algunas áreas de transformación quística que asemejaban quistes aneurismáticos. El diagnóstico anatomopatológico fue condroblastoma de escafoides carpiano.

Tras el tratamiento quirúrgico se inmovilizó la muñeca con una férula antebraquial durante 3 semanas y posteriormente la paciente fue remitida al Servicio de Rehabilitación. En la revisión efectuada a los 3 meses se apreció una recuperación completa de la movilidad de la muñeca e imagen radiológica de consolidación ósea del autoinjerto de cresta iliaca.

Dos años después del tratamiento quirúrgico no hay evidencia de recidiva y persiste una desaparición completa del dolor con movilidad de la muñeca similar a la contralateral, así como una imagen radiológica del escafoides totalmente normalizada (Fig. 4).

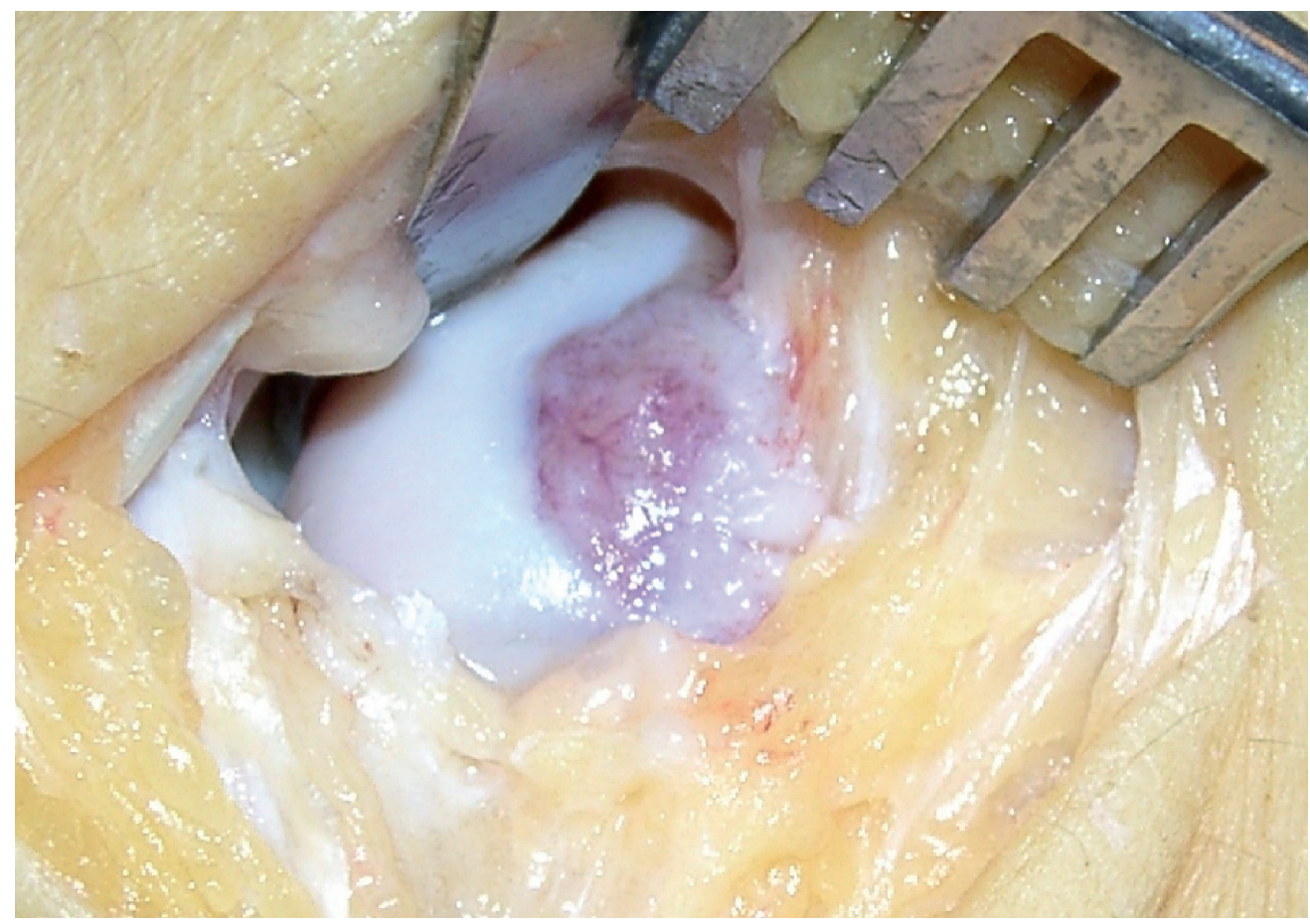

Figura 2. Imagen macroscópica de la lesión. 


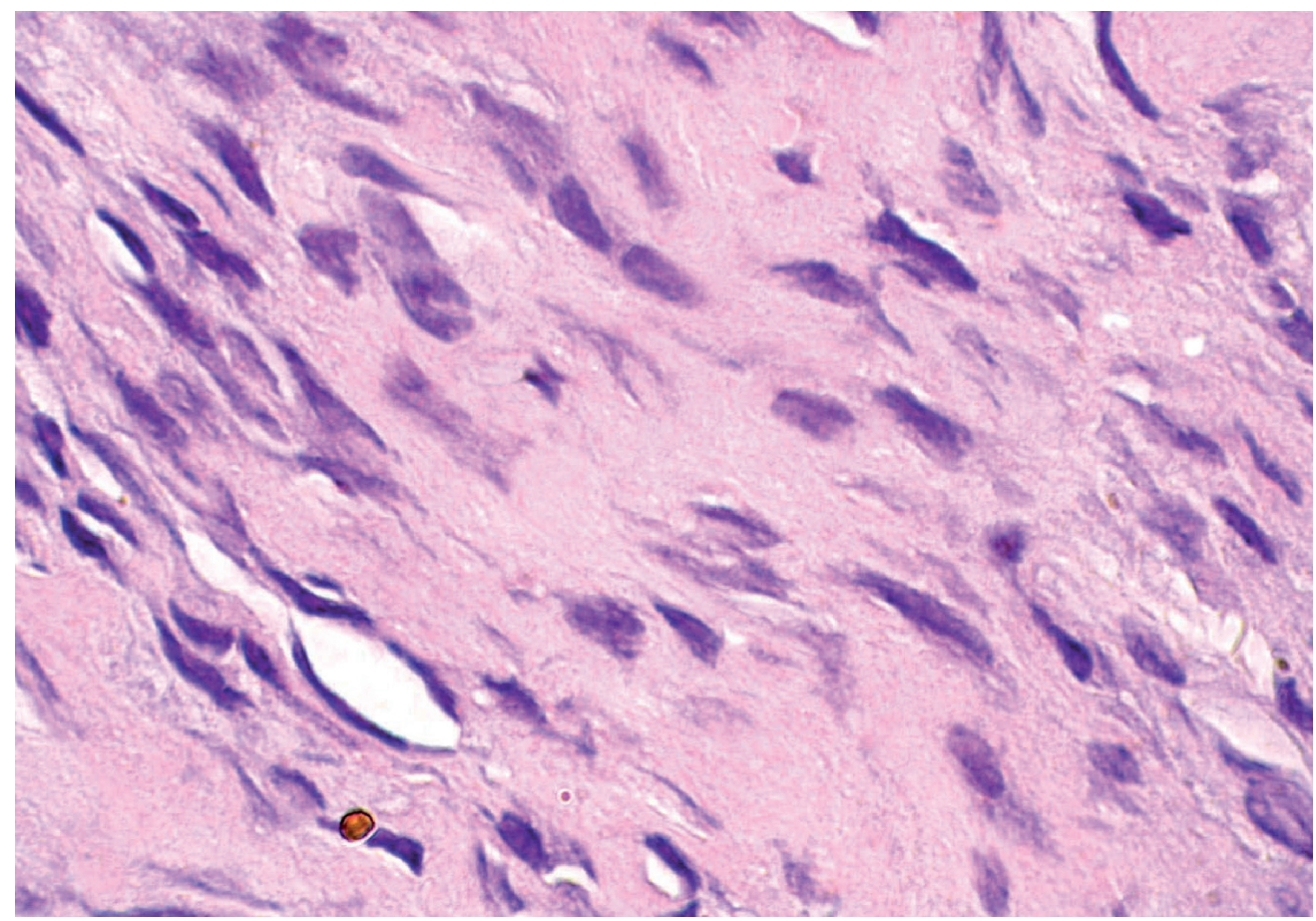

Figura 3. Células pseudocondroblásticas redondeadas u ovoides (HE x 600).

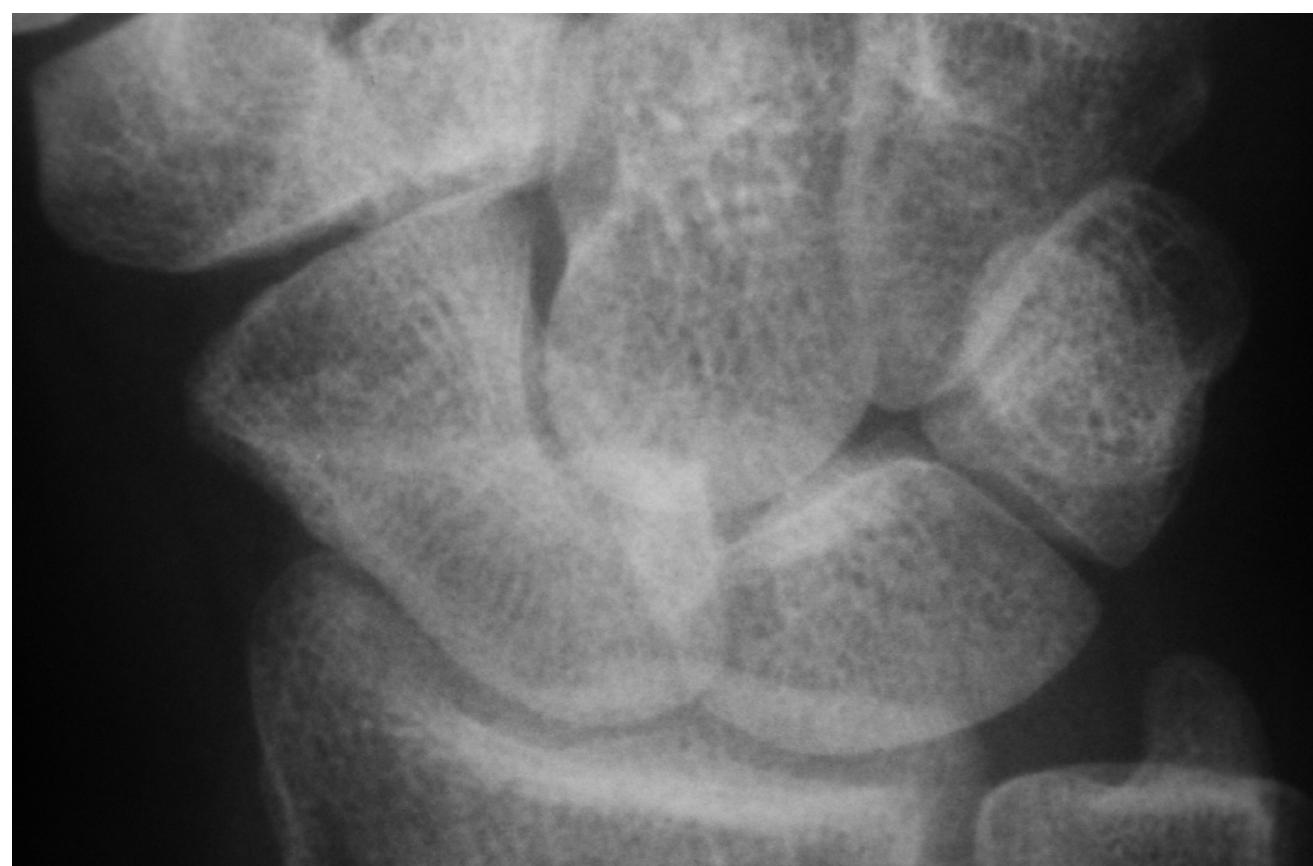

Figura 4. Imagen radiológica a los 2 años del tratamiento. 


\section{DISCUSIÓN}

El condroblastoma es un tumor benigno relativamente raro, cuya localización está relacionada con el cartílago de crecimiento de un núcleo epifisario primario o accesorio $^{2}$. Los sitios más frecuentemente afectados por este tumor son las epífisis de los huesos largos mientras que una minoría afecta a huesos planos y a los huesos de la mano y del pie, con especial afinidad por el calcáneo y el astrágalo ${ }^{3,6}$.

$\mathrm{Su}$ asiento en los huesos del carpo es muy poco frecuente. Sólo se citan tres casos en la revisión de 458 pacientes realizada por Huvos y Marcove ${ }^{4}$, en la que no se especifica qué hueso del carpo está afectado y ninguno entre los 70 casos revisados por Springfield y $\mathrm{col}^{7}$. En la búsqueda realizada en la base de datos Medline sólo encontramos un caso de afectación del escafoides del carpo ${ }^{8}$.

El condroblastoma es dos veces más frecuente en hombres y la mayoría de los pacientes tienen menos de 30 años, con un pico de incidencia en la segunda década de la vida ${ }^{2,6}$, dato que se corresponde con la edad que tenía la paciente que presentamos al comienzo de la sintomatología. Ésta fue inespecífica como en la mayoría de los condroblastomas ${ }^{7}$ y consistió en dolor crónico cercano a la articulación de la muñeca. No se apreció masa de tamaño creciente ni derrame articular como se ha descrito en otros $\operatorname{casos}^{7,8}$.

Las causas de dolor o molestias en la muñeca son múltiples. Las más frecuentes: tenosinovitis, neuropatías por atrapamiento, lesiones ligamentosas y del fibrocartílago triangular, transtornos osteoartríticos, osteonecrosis (enfermedad de Kiemböck) y tumores. Entre éstos son mucho más frecuentes los de partes blandas, especialmente los gangliones en mujeres jóvenes.

En cuanto al diagnóstico diferencial radiográfico de una lesión lítica, bien definida, sin expansión de la cortical y localizada en el carpo, hay que tener en cuenta otros tumores óseos benignos más frecuentes, dentro de su rareza, como el encondroma y el ganglión intraóseo.

La RM es la prueba de elección en la evaluación de tumores en el área de la muñeca. En nuestro caso las características de imagen (Rx, TAC y RM) fueron similares a las descritas habitualmente en otras localizaciones más habituales de tumores benignos condroides $s^{1,2,4,7-10}$.

Los rasgos histológicos eran los descritos típicamente en el condroblastoma: nódulos de matriz cartilaginosa rodeados por un tejido de alta celularidad, relativamente indiferenciado, constituido por células redondeadas u ovoides semejantes a los condroblastos y por células gigantes multinucleadas de tipo osteoclástico aisladas o en grupos, así como pequeñas zonas de calcificación focal ${ }^{2,7}$.

El legrado intracavitario con relleno de autoinjerto óseo procedente de cresta iliaca es el tratamiento de elección ${ }^{2,4,7}$. El comportamiento de estas lesiones es benigno en la gran mayoría de los casos, pero hay descritos casos aislados de condroblastoma con transformación maligna espontánea ${ }^{5}$.

\section{BIBLIOGRAFÍA}

1. JAFFE HL, Lichtenstein L. Benign chondroblastoma of bone: Reinterpretation of socalled calcifying or chondromatous giant cell tumor. Am J Pathol 1942; 18: 969-991.

2. Schajowicz F, Gallardo H. Epiphysial chondroblastoma of bone: a clinico pathological study of sixty-nine cases. J Bone Joint Surg 1970; 52B: 205-226.

3. Kricun ME, Kricun R, Haskin ME. Chondroblastoma of the calcaneus: Radiographic features with emphasis on location. Am J Roentgenol 1977; 128: 613-616.

4. Huvos AG, Marcove RC. Chondroblastoma of bone. A critical review. Clin Orthop 1973; 95: 300-312.

5. Pösl M, Werner M, Amling M, Ritzel $\mathrm{H}$, DELLING G. Malignant transformation of chondroblastoma. Histopathology 1996; 29: 477-480.

6. Bliss DG, MANN RJ. Chondroblastoma of a metacarpal. Report of a case and review of the literature. Clin Orthop Relat Res 1985; 194: 211-213.

7. SPRINGFIELD DS, CAPANNA R, GHERlinZONI F, PIZZI $\mathrm{P}$, CAMPANACCI M. Chondroblastoma. A review of seventy cases. J Bone Joint Surg 1985; 67A: 748-755. 
8. NG JP, LEE JC. Test and teach. Number Sixtytwo. Chondroblastoma of the scaphoid. Pathology 1990; 22: 10, 49.

9. Kroon HM, Bloem JL, Holscher HC, van der Woude HJ, ReIJnierse M, TAMiniau AHM. MR imaging of edema accompanying bening and malignant bone tumors. Skeletal radiol 1994; 23: 261-269.

10. Weatherall PT, Maale GE, Mendelsohn DB, Sherry CS, ERdman WE, PAscoe HR. Chondroblastoma: classic and confusing appearance at MR imaging. Radiology 1994; 190: 467-474. 